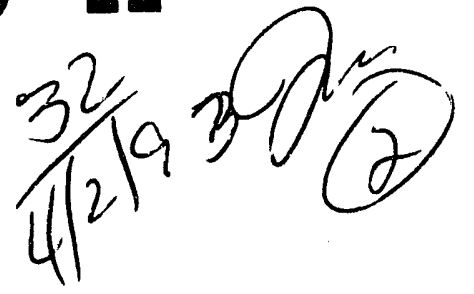

\author{
FNAL-TM- -1828
}

DE93 010525

\title{
Longitudinal Information and Radiation Damage in EM Calorimetry
}

\author{
Dan Green \\ Fermi National Accelerator Laboratory \\ P.O. Box 500, Batavia, Illinois 60510
}

\section{DISCLAIMER}

This report was prepared as an account of work sponsored by an agency of the United States Government. Neither the United States Government nor any agency thereof, nor any of their employees, makes any warranty, express or implied, or assumes any legal liability or responsibility for the accuracy, completeness, or usefulness of any information, apparatus, product, or process disclosed, or represents that its use would not infringe privately owned rights. Referprocess discosed, or recific commercial product, process, or service by trade name, trademark, manufacturer, or otherwise does not necessarily constitute or imply its endorsement, recommentation and opinions of authors expressed herein do not necessarily state or reflect those of the United States Government or any agency thereof.

February 1993

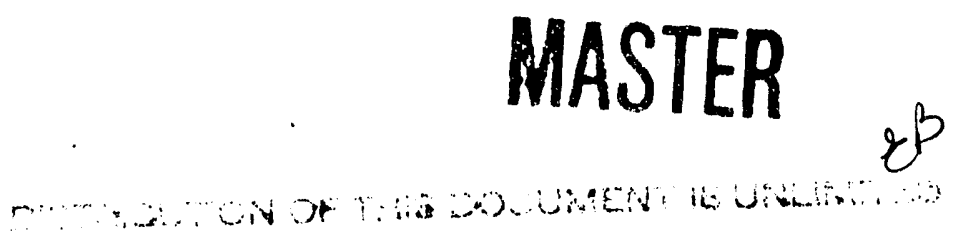




\section{Disclaimer}

This report was prepared as an account of work sponsored by an agency of the United States Government. Neither the United States Government nor any agencv thereof, nor any of their employees, makes any warranty, express or implied, or assumes any legal liability or responsibility for the accuracy, completeness, or usefulness of any information, apparatus, product, or process disclosed, or represents that its use would not infringe privately owned rights. Reference herein to any specific commercial product, process, or service by trade name, trademark, manufacturer, or otherwise, does not necessarily constitute or imply its endorsement, recommendation, or favoring by the United States Government or any agency thereof. The views and opinions of authors expressed herein do not necessarily state or reflect those of the United States Government or any agency thereof. 


\title{
LONGITUDINAL INFORMATION AND RADIATION DAMAGE IN EM CALORIMETRY
}

\author{
Dan Green \\ Fermi National Accelerator Laboratory \\ Batavia, Illinois
}

February 5, 1993

1. Introduction

The SSC radiation field is higher than that encountered by previous hadron collider detectors [1]. In particular, the electromagnetic (EM) calorimeter compartment sees the highest radiation dose. Since an EM calorimeter also makes the most precise energy measurement, special care must be lavished on this part of a calorimeter. Previous studies have concentrated on Monte Carlo examinations of 2 longitudinal compartments within the EM which can alleviate radiation damage [2]. Recently, it was realized that a "shower maximum" detector, such as exists in $\mathrm{CDF}$, also contains information of the conversion point of an electromagnetic shower. As such, it can potentially be used in a fashion analogous to the longitudinal compartments [3], although it is not designed to be optimized for this role.

\section{Damage Profile}

The damage in the EM compartment is thought to be induced by the interactions themselves. Therefore, the dose energy profile is expected to follow that of a peripheral neutral pion. In order to approximate that profile, $10 \mathrm{GeV}$ electrons incident on the "hanging file" (HF) test calorimeter [4] were used. This device consisting, in this incarnation, of 40 plates of $1 / 8$ " $\mathrm{Pb}$ followed by 55 plates of 1 " $\mathrm{Fe}$ is a reasonable approximation to the longitudinal structure of the SDC calorimeters [1].

The average longitudinal energy profile, integrated over many incident electrons, was assumed to represent the dose profile at the SSC. The relationship of dose to "damage" (loss of light output) was assumed to be linear. The assumption of linearity has been checked experimentally [5]. Therefore, the assumed depth (z) distribution of the response of a radiation damaged EM calorimeter can be written;

$$
1-d(z)=1-a E(z)
$$

where $\mathrm{d}$ is the fractional light loss (damage), $\mathrm{E}(\mathrm{z})$ is the energy profile of $10 \mathrm{GeV}$ electrons, and $\mathrm{a}$ is a normalization factor. The function $1-\mathrm{d}(\mathrm{z})$ for a maximum light loss of $50 \%$ is shown in Fig. 1. The peak damage occurs at $\sim$ plate $\# 10$ or $\sim 5$ radiation lengths $(\mathrm{Xo})$.

Electrons incident on the $\mathrm{HF}$ at $170 \mathrm{GeV}$ were used to study the effects of radiation damage on the response of the EM calorimeter. The HF data consisted of the readout of a scintillator behind each plate. Therefore, the damaged calorimeter could be simulated by multiplying each plate response by $1-d(z)$ and then summing all plates. The result of that 
exercise is shown in Fig. 2. The EM calorimeter response is characterized by the mean and second moment ( $\mathrm{rms}$ ) of the output energy distribution. The mean energy falls roughly linearly with maximum damage, dmax. The drop is $\sim 30 \%$ for a $50 \%$ peak fractional light 10 s.

The HF data had an undamaged rms of $1.5 \%$. The rms for each dmax had this undamaged rms unfolded in quadrature to yield the "induced constant term" in the EM energy" resolution. That quantity is plotted in Fig. $2 \mathrm{~b}$ as a function of dmax. The relationship is roughly linear, and is about $2.5 \%$ at $50 \%$ peak fractional light loss. Requiring an induced $\mathrm{rms}<1 \%$ means that the value for dmax must be $<20 \%$ if no corrections are applied.

\section{E1/E2, Esm and the Induced Constant Term}

The radiation damage creates an inhomogeneous calorimeter. Fluctuations in shower development then lead to errors in energy measurement. Since the main longitudinal fluctuation in an EM shower is simply the conversion point, measurements of that conversion point allow one to partially correct for induced inhomogeneities [2].

The HF data was used to provide experimental confirmation of the conclusions of previous Monte Carlo studies. A front longitudinal compartment of 20 plates, 10 Xo, was "constructed". The ratio of the energy in that compartment, E1, and the energy in the remainder of the calorimeter, E2, was used as a measure of the longitudinal development of the EM shower. The correlation between the total e zergy output of the calorimeter, Eem, and the ratio E1/E2 is shown in Fig. 3 for various $(0.1,0.3,0.5,0.7)$ values of dmax. Clearly, there is a correlation between these 2 quantities.

Similarly, a sampling of the shower at fixed depth contains information on the shower development. 4 sample at plate \#9 $(\sim 4.5 \mathrm{Xo})$ was defined to be the "shower maximum" energy, Esm. The correlation between Esm and the total calorimeter energy is shown in Fig. 4 for various values of dmax. Again, there is a correlation, as intuition suggests. Note that, at least for this depth (10 Xo vs 4.5 Xo respectively), the E1/E2 ratio is better correlated with Eem than is Esm. In both cases, the correlation increases with peak damage as expected.

The simplest linear correction to Eem was applied to the data, using the correlations observed in Fig. 3 and Fig. 4.

$$
\begin{aligned}
& E^{\prime}=E+b(E 1 / E 2-c) \\
& E^{\prime \prime}=E+g(E s m-h)
\end{aligned}
$$

The resulting induced constant term in the energy resolution is shown in Fig. 5 using the E1/E2 ratio. The uncorrected data of Fig. 2 is also shown as a function of dmax. Clearly, one can reduce the degradation due to radiation damage by a factor $\sim 2$. This experimental result confirms the Monte Carlo study [2]. The analogous results using Esm to correct are shown in Fig. 6. Again, there is an improvement, but it appears to be less than that afforded by E1/E2. As will be seen, the poorer correction is the result of the choice of depth of the shower maximum sample. That depth is chosen to optimize the physics performance of the shower maximum detector. If the E1/E2 boundary were chosen to coincide with the location of the shower maximum sample, Fig. 5 and Fig. 6 would be very similar.

In any case, if a shower maximum detector exists in the absence of explicit longitudinal segmentation, then it may be used to reduce the effects of radiation damage. One might question if the counting statistics are sufficient. The shower at maximum has $\sim 10$ 
$\mathrm{mip} / \mathrm{GeV}$. For a transducer with $2 \mathrm{pe} / \mathrm{mip}$ [1], the statistics at $10 \mathrm{GeV}$ (a minimum in the SSC enviroment [1]) is defined by $>200$ pe. Therefore, the value of Esm was smeared by a Gaussian with a conservative value of $10 \% \mathrm{mms}$ before the correction shown in Eq. 2 was applied. The results are also shown in Fig. 6 . Clearly, the photostatistics do not degrade the correction algorithm to the total energy significantly.

\section{E1-E2 Boundary Optimization}

The EM showers of interest at the SSC are higher energy than the "minbias" background which is responsible for the radiation damage. However, the shower maximum detector is most useful for fairly low energies [1]. The.efore, that deiector is placed at a fairly shallow depth. This depth is, unfortunately, not optimal for protection against the effects of radiation damage.

The HF data was used to "construct" EM calorimeters with 2 depth segments. The boundary between depth segments was varied, and the optimal depth for the boundary with respect to the radiation damage correction was sought. The scatter plots for Eem vs E1/E2 for E1-E2 boundaries of $10,15,20$, and 25 plates $(\sim 5,7.5,10$, and $12.5 \mathrm{Xo})$ are shown in Fig. 7 for a damage of, dmax $=0.5$. Clearly, a boundary at the shower maximum depth location is not optimal for the correction of radiation damage effects. For the higher energy electrons, $E=170 \mathrm{GeV}$, the boundary should be deeper in the EM calorimeter.

The data were corrected as in Eq. 2 for several E1-E2 boundaries. The resulting induced constant term in the energy resolution for $\mathrm{dmax}=0.5$ is shown as a function of the location of the 1-2 compartment boundary in Fig. 8. Note that the datum at plate\# $=0$ corresponds to no correction, as in Fig. 2. Clearly, there is a broad minimum. At the shower maximum depth (plate \# 9), the induced constant term is almost twice the minimum value. Hence, the comparison of Fig. 5 with Fig 6 . At the same depth E1/E2 and Esm corrections contain essentially the same information, and have roughly the same utility. The physics goals of the shower maximum detector dictate that it be placed at a shallow depth [1]. The reduction of the degradation due to radiation damage, see Fig. 8, dictate a depth boundary at $\sim 10$ Xo. If explicit depth compartments are to be provided in addition to the shower maximum sample, then the boundary between them should be chosen $\sim$ twice as deep as the shower maximum detector, where the ratio E1/E2 is of order 1.

\section{References}

1. SDC Technical Design Report, SDC-92-101 (1992).

2. D. Green, A. Para and J. Hauptman "Radiation Damage, Calibration and Depth Segmentation in Calorimeters", Fermilab-FN-565, May 1991.

3. A. Beretvas, D. Green, J. Marraffino and W. Wu, "Use of a Shower Maximum Detector to Reduce Radiation Damage Sensitivity in EM Calorimetry", Fermilab-FN600, December 1992.

4. A. Beretvas, et al., "Beam Tests of Composite Calorimeter Configurations from Reconfigurable-Stack Calorimeter", accepted by Nuc. Inst. Meth.

5. L. Hu, et al., "Radiation Damage of Tile/Fiber Scintillator Modules for the SDC Calorimeter", Fermilab-TM-1769, 1991. 


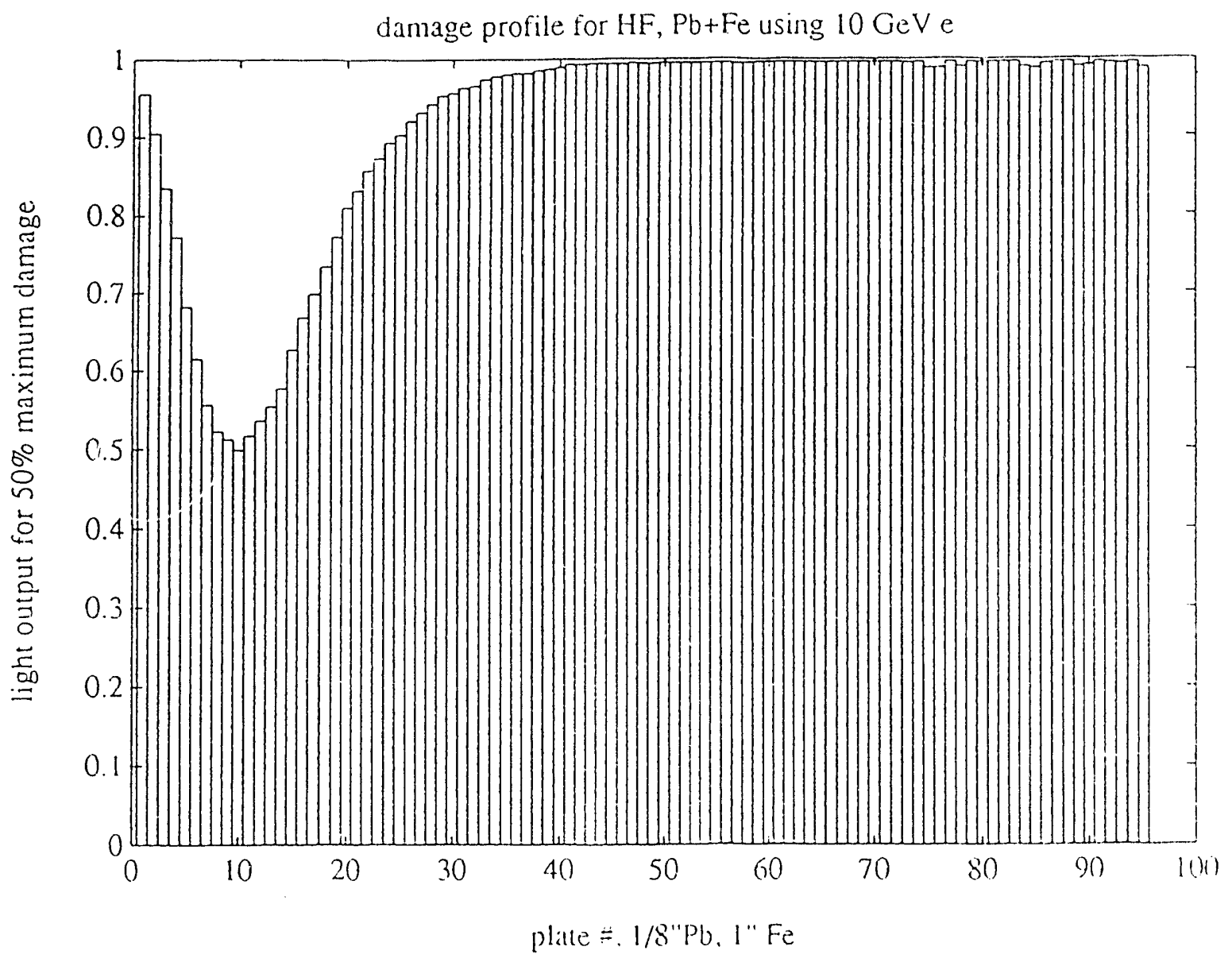

Fig. 1 The "damage profile" for the HF calorimeter. Data from the $10 \mathrm{GeV}$ e sample were used and normalized to $50 \%$ maximum light output loss. The HF consists of 40 plates of $1 / 8$ " $\mathrm{Pb}$ and 55 plates of 1 " Fe. 

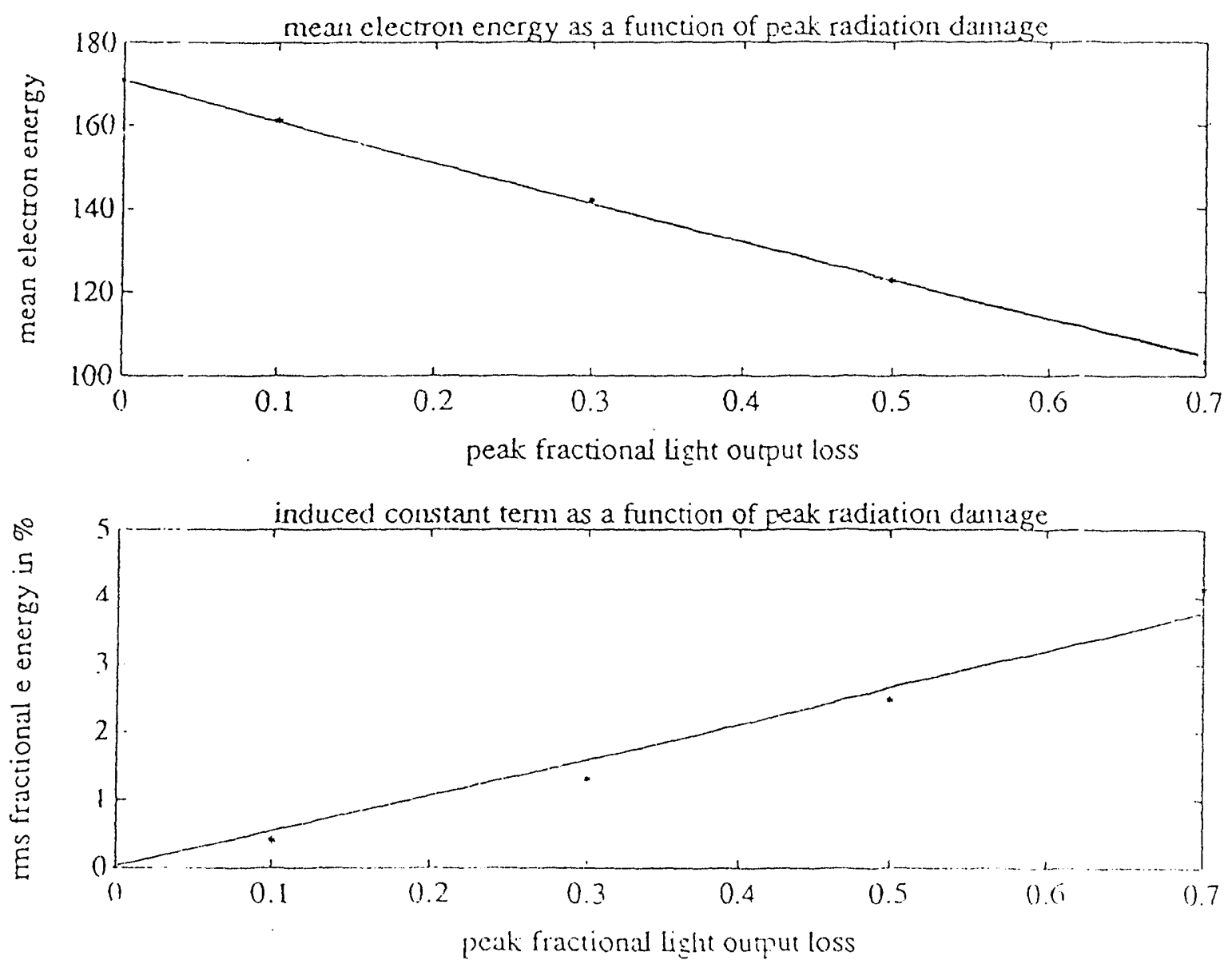

Fig. 2 The effect of radiation damage on the $\mathrm{HF}$ response to $170 \mathrm{GeV}$ electrons.

a. Mean electron energy as a function of maximum fractional light loss, $<\mathrm{E}(\mathrm{dmax})\rangle$.

b. Induced constant term as a function of maximum fractional light loss, $\sqrt{r m s(E(d \max ))^{2}+r m s(E(0))^{2}}$.

The lines are a rough approximation to the observed behavior. 

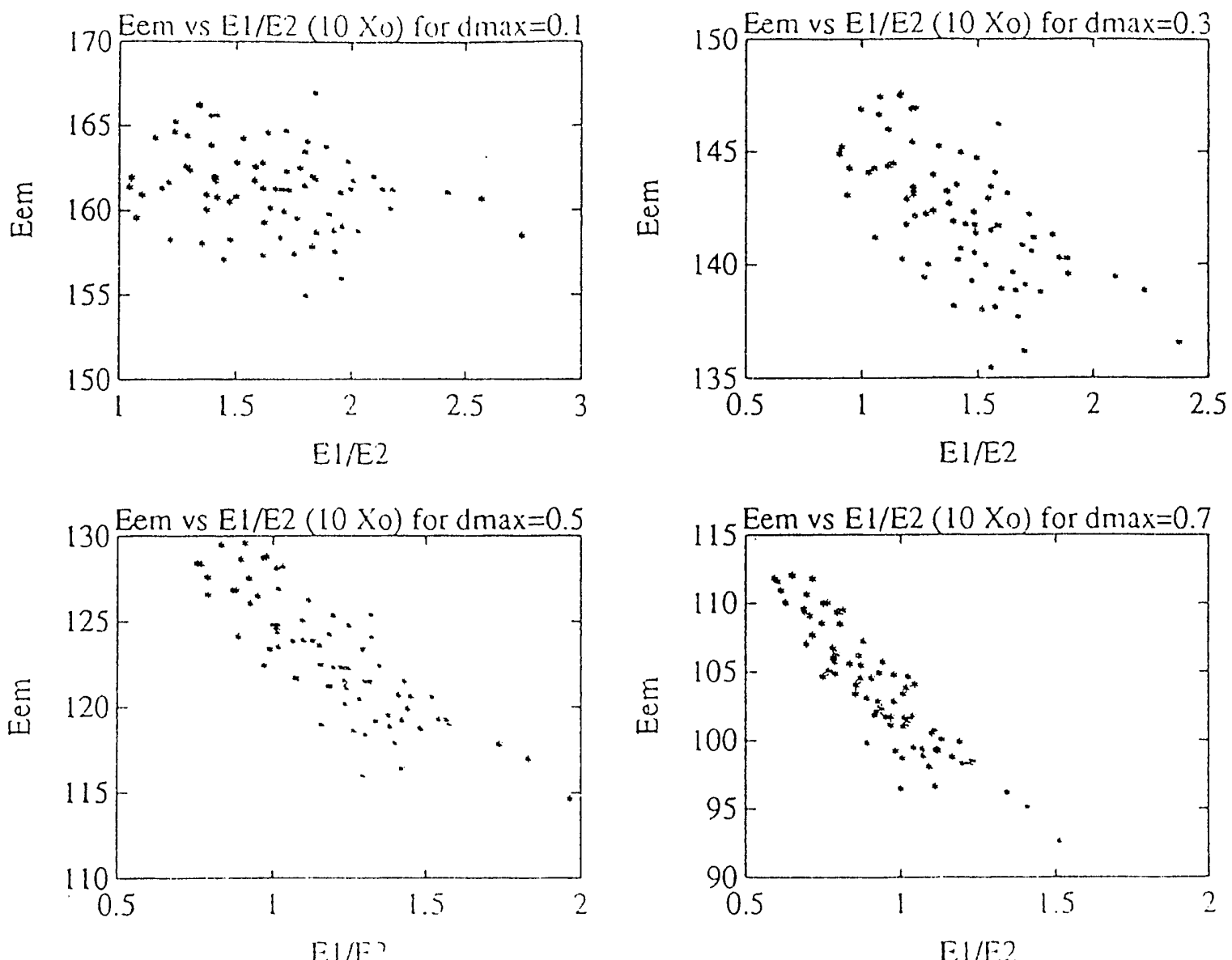

Fig. 3 Energy for $170 \mathrm{GeV}$ electrons incident on the $\mathrm{HF}$ as a function of the ratio of energy in the first compartment, (20 plates $\sim 10$ radiation lengths) to the second compartment (remainder of $\mathrm{HF}$ ).
a. maximum fractional light loss, $\mathrm{dmax}=0.1$.
b. $d \max =0.3$
c. $\operatorname{dmax}=0.5$
d. $\operatorname{dmax}=0.7$ 

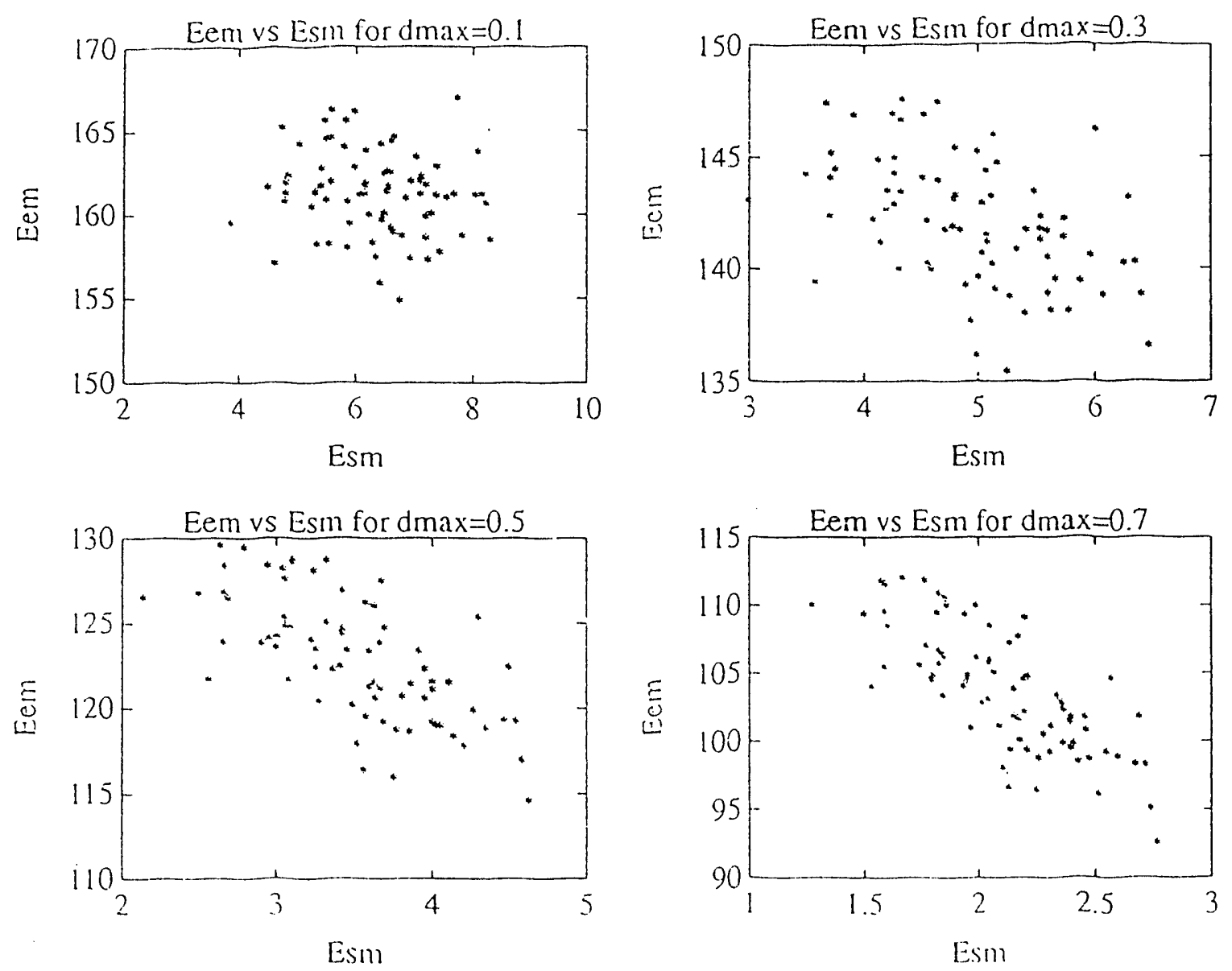

Fig. 4 Energy for $170 \mathrm{GeV}$ electrons incident on the $\mathrm{HF}$ as a function of the energy at plate 9 ( Esm $=$ "shower maximum" energy).
a. maximum fractional light loss, dmax $=0.1$
b. $\operatorname{dmax}=0.3$
c. $\operatorname{dmax}=0.5$
d. $\operatorname{dmax}=0.7$ 


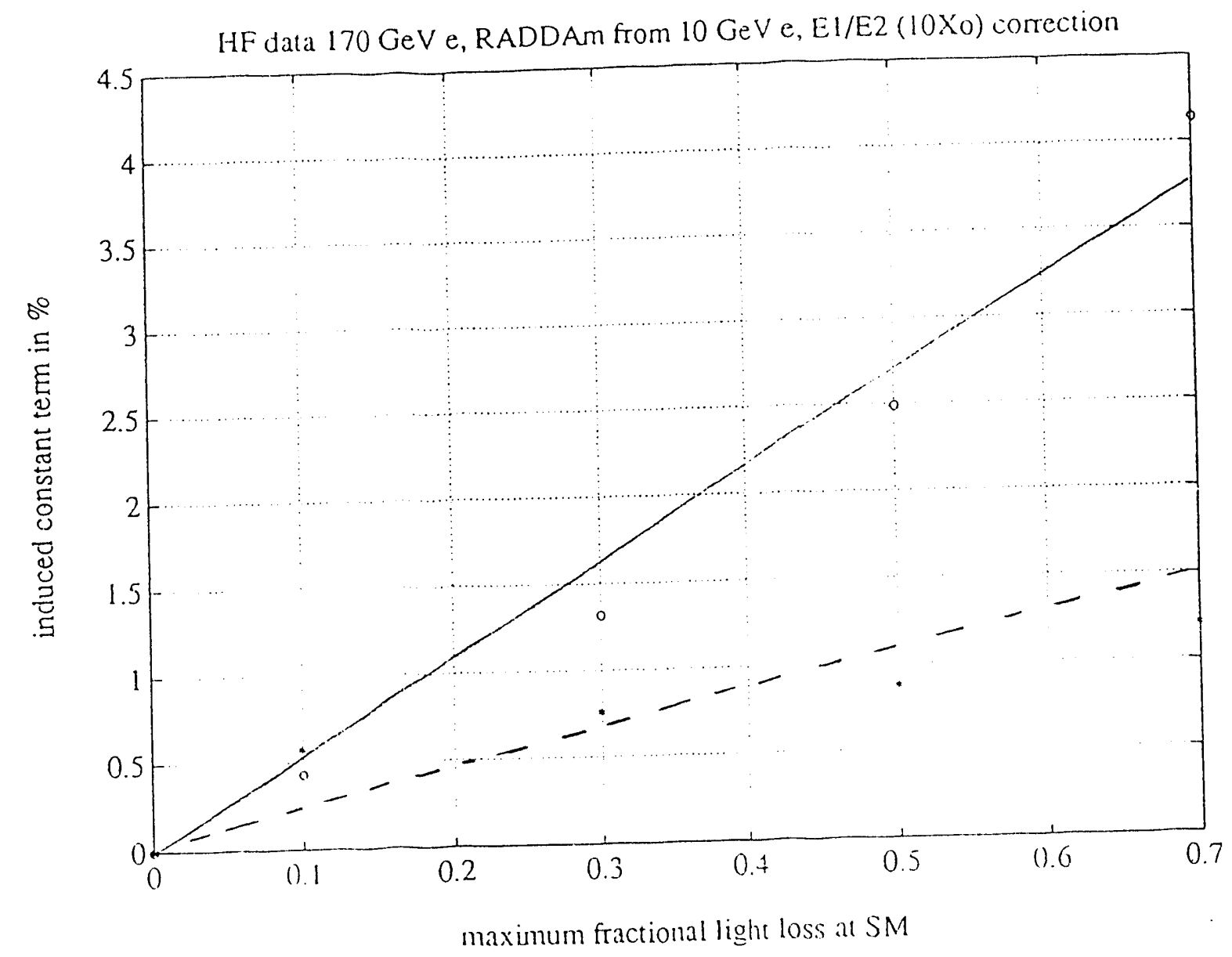

Fig. 5 Induced constant term in the energy resolution for $170 \mathrm{GeV}$ electrons incident on the $\mathrm{HF}$ as a function of the maximum fractional light loss, dmax. The lines are added to guide the eye. The full line represents the constant term without correction, $\sqrt{r m s(E(d \max ))^{2}+r m s(E(0))^{2}}$. The dashed line is the induced constant term after correction to $E$ using E1/E2. 


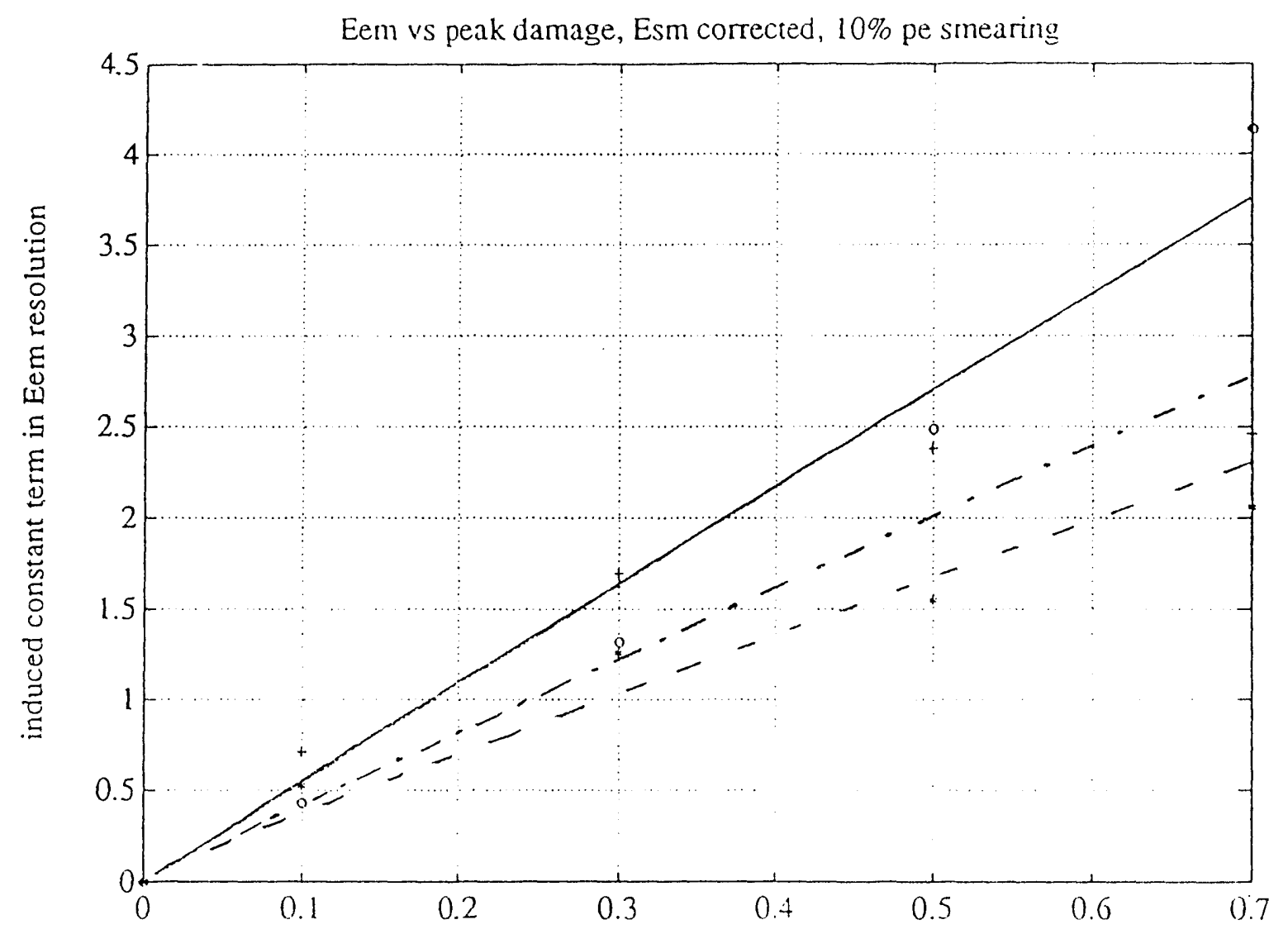

peak fractional light output loss at $S M$

Fig. 6 Induced constant term in the energy resolution for $170 \mathrm{GeV}$ electrons incident on the HF as a function of the maximum fractional light loss, dmax. The lines are added to guide the eye. The full line represents the constant term without correction, $\sqrt{r m s(E(d \max ))^{2}+r m s(E(0))^{2}}$. The dashed line is the induced constant term after correction to $\mathrm{E}$ using Esm. The dot-dashed line represents the results after a $10 \%$ Gaussian smearing is applied to Esm which represents the worst case photoelectron statistical error. 

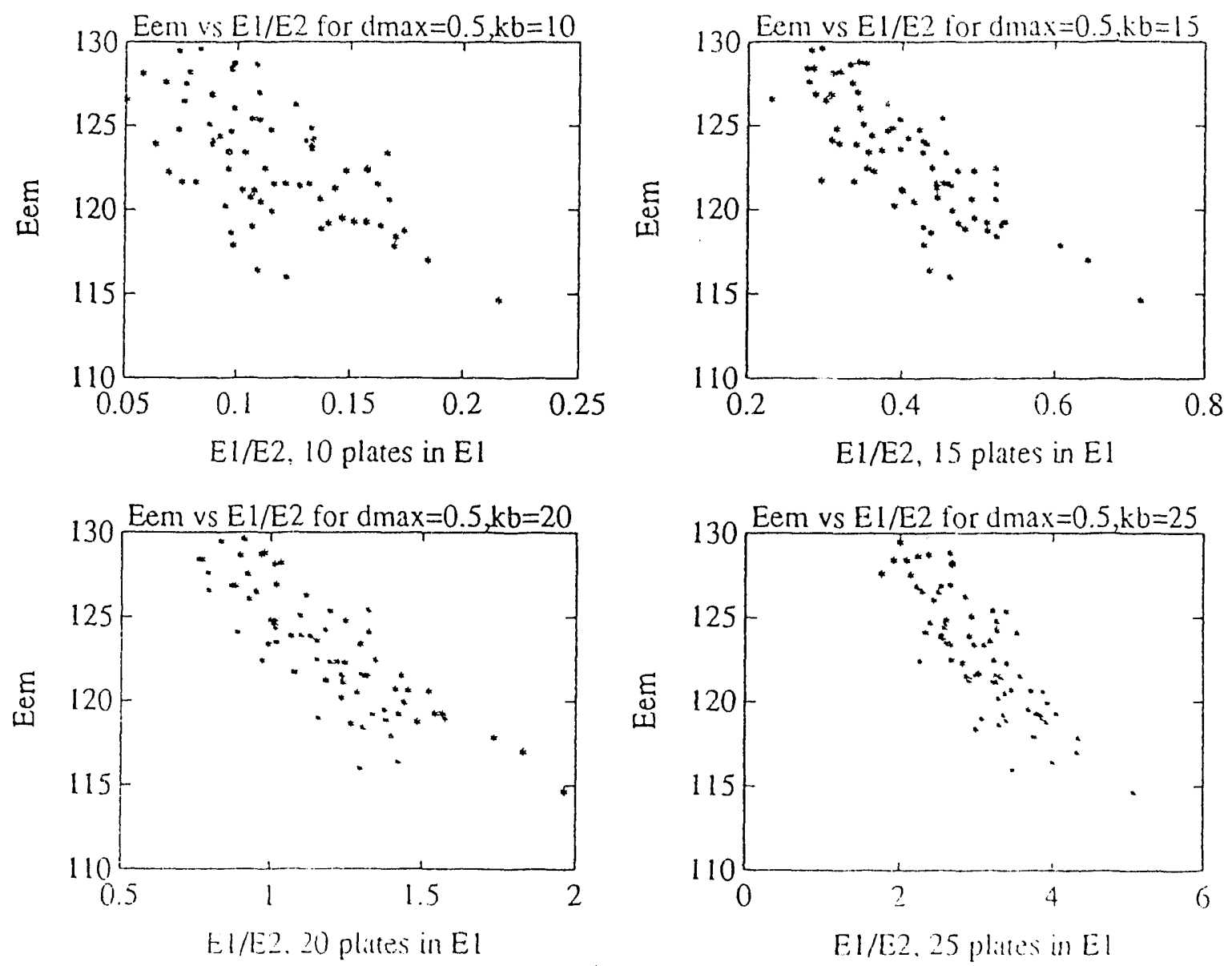

Fig. 7 Energy for $170 \mathrm{GeV}$ electrons incident on the $\mathrm{HF}$ as a function of the ratio of energies in the front compartment, E1, to the rear compartment, E2 with dmax = 0.5 .
a. compartment 1 is 10 plates, $\sim 5$ Xo deep
b. compartment 1 is 15 plates, $\sim 7.5$ Xo deep
c. compartment 1 is 20 plates, $\sim 10$ Xo deep
d. compartment 1 is 25 plates, $\sim 12.5$ Xo deep 


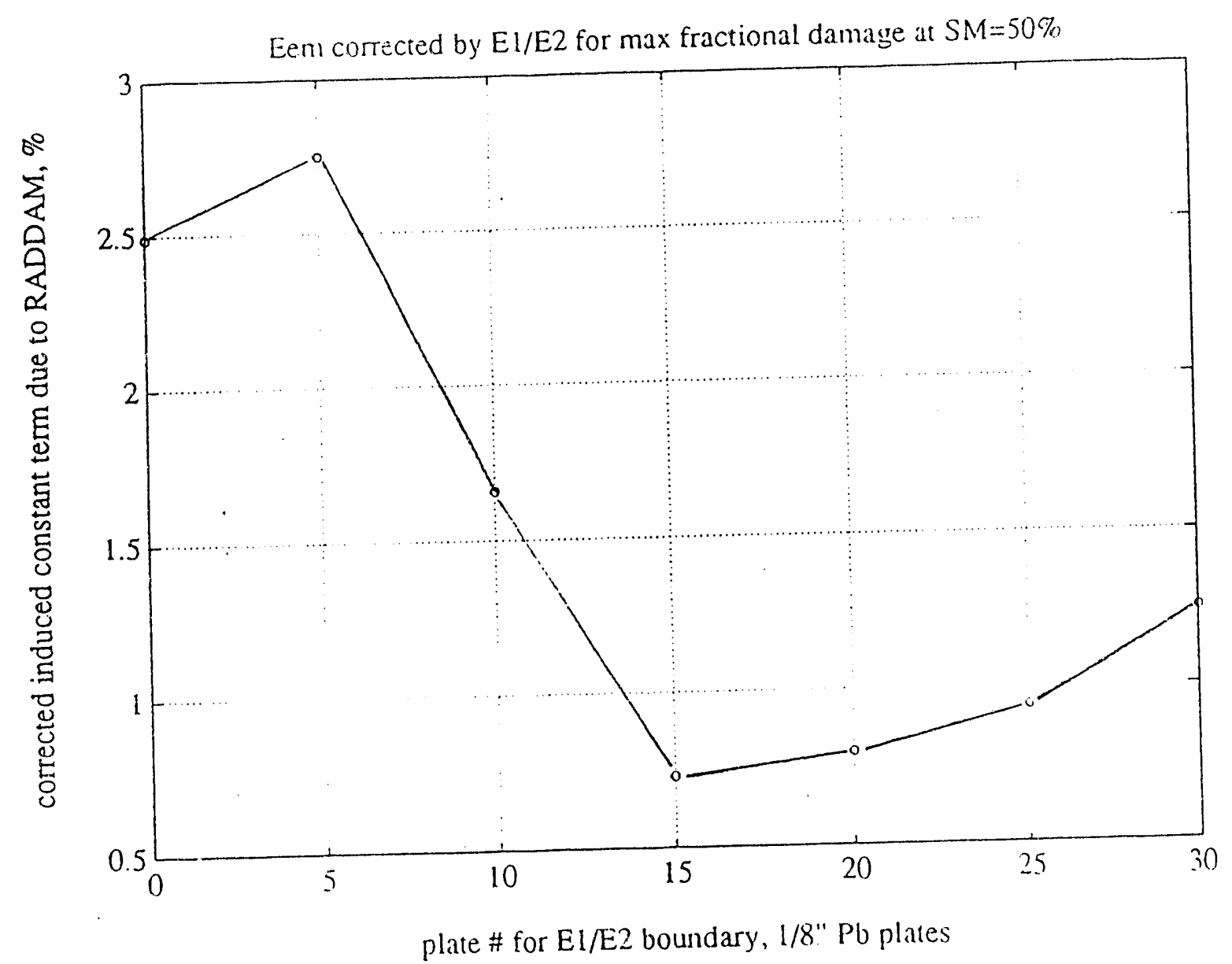

Fig. 8 Induced constant term in the energy resolution for $170 \mathrm{GeV}$ electrons incident on the $\mathrm{HF}$ as a function of the depth of the first longitudinal EM compartment. The induced constant term has been corrected linearly using the ratio E1/E2. The maximum fractional light loss is dmax $=0.5$. The optimal depth for the E1-E2 boundary is $7-10$ radiation lengths, Xo. 

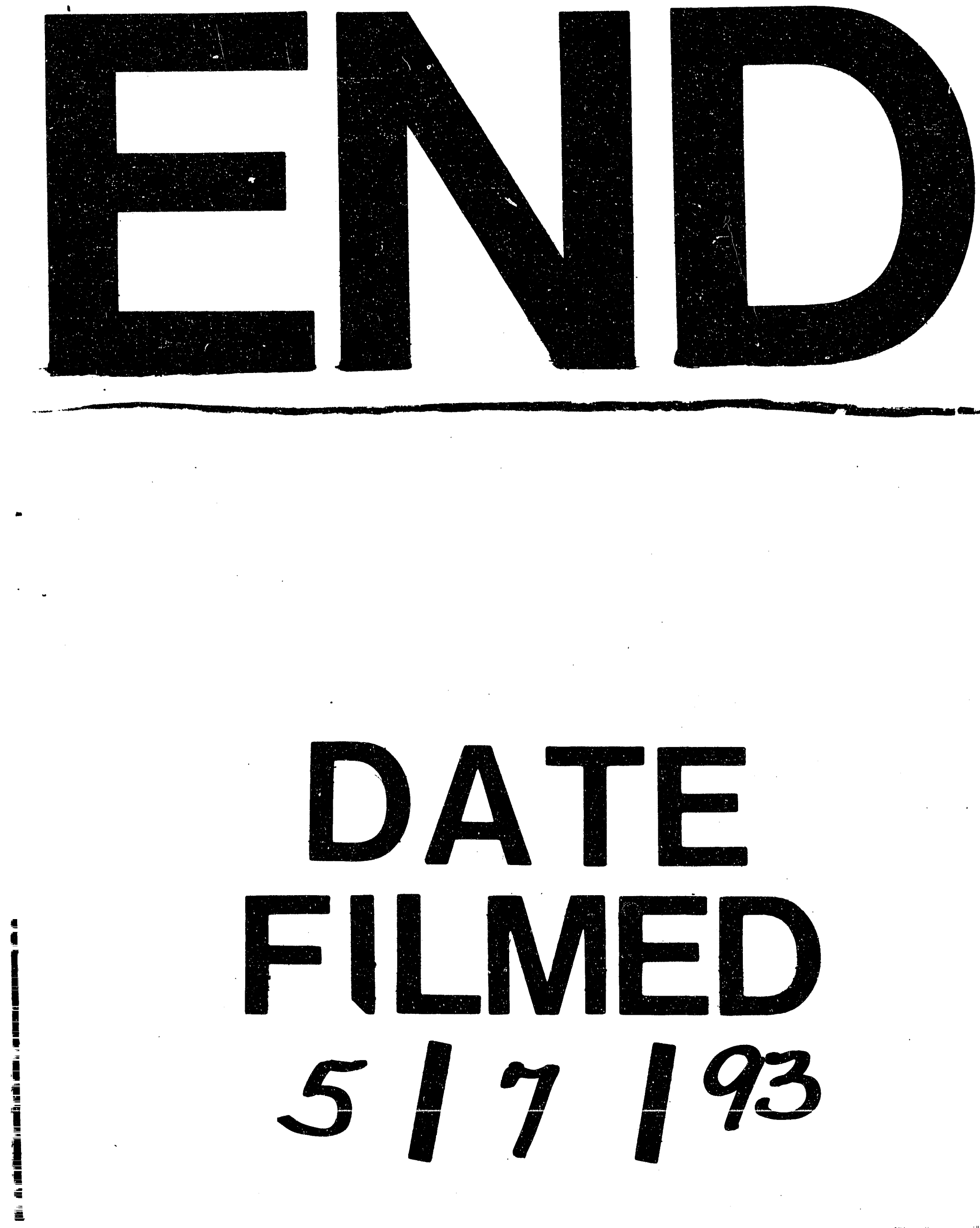
, , , , , , , , , ,

. . .

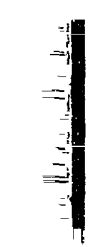

